\title{
Microwave Assisted Healing of Thermally Mendable Composites
}

\author{
Edward D. Sosa, ${ }^{1}$ Thomas K. Darlington, ${ }^{2}$ Brian A. Hanos, ${ }^{2}$ and Mary Jane E. O'Rourke ${ }^{3}$ \\ ${ }^{1}$ ERC, Houston, TX 77058, USA \\ ${ }^{2}$ Nano Composix, San Diego, CA 92111, USA \\ ${ }^{3}$ NASA Johnson Space Center, Houston, TX 77058, USA
}

Correspondence should be addressed to Edward D. Sosa; edward.sosa-1@nasa.gov

Received 19 September 2014; Accepted 14 January 2015

Academic Editor: Kelvin Yeung

Copyright (C) 2015 Edward D. Sosa et al. This is an open access article distributed under the Creative Commons Attribution License, which permits unrestricted use, distribution, and reproduction in any medium, provided the original work is properly cited.

\begin{abstract}
Polymer matrix composites offer high specific strength; however, their potential weight savings have been limited by the concern of damage tolerance. If microcracking and similar incurred damage could be autonomously sealed, composite structures could be built thinner and lighter while still addressing damage tolerance, thus achieving the weight savings they promise. Various selfhealing mechanisms have been proposed to this end. Herein, a method of thermally reversible polymerization is investigated. To date, thermally activated repair of composites have been accomplished typically through resistive heating, which has certain inherent complexities. An alternate heating method, via microwave exposure of carbon nanotubes incorporated throughout a thermal reversible polymer matrix, is demonstrated. Carbon nanotube-doped composites exhibit enhanced microwave absorption over an undoped control sample. Furthermore, it is shown that these composites can be heated locally by a focused microwave source. The particular composite formulation and layup studied could be uniformly heated to the targeted healing temperature of $100^{\circ} \mathrm{C}$ in as little as 20 seconds, followed by a healing time on the scale of minutes with total time depending upon the extent of damage.
\end{abstract}

\section{Introduction}

Advanced composite materials will continue to revolutionize a variety of commercial markets due to their many advantages. Foremost are their lightweight and high strength. Boeing's Dreamliner is one significant achievement for composites' contribution to the advancement of technology, with a composite airframe that makes it Boeing's most energyefficient commercial passenger aircraft in history $[1,2]$. Polymer composites impact other industries in addition to aerospace: energy, transportation, sporting, infrastructure, and military sectors. Other potential applications include wind turbine blades, boat hulls, drones, military vehicles, and vehicles for individual and mass transportation.

Polymer matrix composites have an added benefit of cost reductions in energy, production, and maintenance associated with these structural materials. Their lightweight nature reduces energy expenditure not only for operation of the final structure, but also in the energy required for transportation, maintenance, and installation that occur along the path to the usable end product. The chemical resistance of many polymer systems makes them less susceptible to oxidative corrosion, thereby requiring less servicing over the component lifetime.

Unfortunately, polymer matrix composites have been limited by an integral design concern associated with damage tolerance. Any damage, however small, can result in a decrease of the structural integrity of the material and require costly repair. The concern of barely visible damage in the form of microcracks results in the design of thicker composite structures, which translates to an increase in overall weight. Challenges associated with damage tolerance in polymer matrix composites must be successfully addressed in order to ensure that high structural reliability is coupled with the significant weight savings. The introduction of manufacturing defects such as voids or delamination is another concern that must also be addressed. Self-healing materials provide a viable means to surmount damage tolerance and manufacturing concerns, thereby allowing for the realization of safe and reliable structures with reduced mass. 
The natural healing capability of biological systems has been the inspiration for the development of smart materials that can undergo internal repair. Biological systems have the distinct advantage of self-repair owing to living organisms' ability to adapt to changes in the environment. Replicating this healing property within material systems has been an enormous challenge in materials science. Many self-healing methods have been proposed and can be categorized as exhibiting either intrinsic or extrinsic healing mechanisms [3-5]. Extrinsic mechanisms require healing agents while intrinsic mechanisms possess an inherent property within the material that allows for healing. The most notable extrinsic mechanisms are the encapsulation or vascular network approaches [6-11]. While these mechanisms are autonomous, limitations include the inability to execute more than one healing cycles, reduced structural integrity with high loadings, requirements for proper mixing of curing agents, and challenges to the manufacture of complex parts. The intrinsic approach circumvents these issues; however, it, too, has some limitations. The intrinsic approach accomplishes healing through bond reformation of the polymer system and, in most cases requires an energy input such as heat or light to initiate healing [12-15]. Recently, researchers in Spain have achieved intrinsic healing in a poly (urea-urethane) polymer by pressing the material together at an interface (damage site) and allowing it to stand for 2 hours at room temperature [16]. While the result is impressive, this is an elastomeric system and therefore unsuitable for structural applications. Soft elastomeric materials such as these possess Young's moduli below $50 \mathrm{MPa}$, whereas high performance structural engineered materials require moduli above $1 \mathrm{GPa}$. Other work has focused on the use of thermoplastic or thermoset materials, which are more desirable for structural applications due to their greater stiffness. Many of these systems require exposure to elevated temperatures or to photons to induce repolymerization for composite healing. Thermally activated healing is the preferred method since the structure is exposed to sunlight in many applications, which could lead to softening while is use if photon-induced healing were the selected method. Therefore, composites that undergo thermally reversible polymerization are likely the best selection for smart materials that can provide intrinsic repair.

The Diels-Alder chemistry of maleimide and furan molecules allows for thermally activated healing through thermal reversible covalent bonding between the two chemical moieties. One particular system that has been studied extensively is the bismaleimide tetrafuran (2MEP4F), which undergoes thermally reversible polymerization above $90^{\circ} \mathrm{C}$ and has a Young's Modulus above $4 \mathrm{GPa}[12,17]$. Healing in 2MEP4F composites has been initiated and sustained through resistive heating via an embedded electrical network. While this method of heating is simple and effective, it does not provide the most efficient heating as it requires substantial current to heat the materials to the elevated temperature necessary for repolymerization. There are also potential concerns of discontinuities in the electrical pathway should the network become damaged, or of complexity of the network, should localized heating be desired. If the electrical network consists only of two electrodes, then application of heat by current passing between those two electrodes necessitates that the entire structure is subjected to heating and therefore to softening, which will be unfavorable to a system under load. Localized heating is preferred, which will require complex integrated electrical networks, adding substantial weight and cost to the system. The microwave absorption property of carbon nanotubes (CNTs) provides an alternative mechanism for localized heating, and additionally introduces multifunctional properties. In this case, a compact focused microwave source is a better alternative, as it would allow for heating of only the area of interest, thus not jeopardizing other regions of the structure.

CNTs are known to absorb microwave radiation and readily convert it to heat with extremely high efficiency $[18,19]$. Therefore, by incorporating CNTs into the $2 \mathrm{MEP} 4 \mathrm{~F}$ thermally reversible polymer matrix, these composite can be heated very efficiently through exposure to microwaves. In this report it is demonstrated that $2 \mathrm{MEP} 4 \mathrm{~F}$ composites can be healed by exposure to a microwave source. Depending on the extent of damage, panels can be healed in a little as 3 minutes of heating at the required temperature of repolymerization. Composite panels containing CNTs exhibit greater heating and healing efficiencies relative to panels without CNTs. These panels can be heated uniformly across the entire test specimen when completely exposed to microwaves, and local heating can be accomplished with the use of a focused microwave generator. The use of a focused microwave source can heat the composites to the targeted healing temperature of $100^{\circ} \mathrm{C}$ in as little as 20 seconds.

\section{Experimental}

Composite panels were fabricated using Hexcel plain weave HexForce Carbon Fabric cut in $10 \mathrm{~cm}^{2}$ sheets such that a $\left[0^{\circ}, 60^{\circ},-60^{\circ}\right]_{2 s}$ orientation lay-up could be achieved. The fibers were coated with multi-wall carbon nanotubes (MWCNTs), single-wall carbon nanotubes (SWCNT), and spun carbon nanotube ribbons drawn from nanotube forests. MWCNTs and SWCNTs were deposited by spray coating. Nanotube powders were dispersed in methanol by probe sonication and subsequently spray coated onto carbon fiber fabrics. Spun CNTs obtained from Solarno were directly deposited onto fibers followed by densification with methanol to improve adhesion to fibers. Panels were fabricated by infiltrating the coated fibers with the $2 \mathrm{MEP} 4 \mathrm{~F}$ resin through either custom resin transfer molding of the 2MEP4F resin [17] in most cases, or by wet lay-up in the case of two panels that had SWCNTs dispersed directly into the 2MEP4F resin.

Two sets of experiments were conducted to demonstrate heating and healing efficacy of composite panels subjected to microwaves. Heating effectiveness was measured by the ability to rapidly and uniformly heat the composite panels to the required temperature by microwave exposure. These experiments were designed to also show that a focused microwave source could be utilized to locally heat such composites. Healing effectiveness experiments were designed to show that CNT introduction into the composites could facilitate improved repair. 
Composite panels were heated using both a custom-modified microwave oven and a custom-built compact focused microwave source. The microwave oven is a Microwave Research and Applications Inc BP-111 Microwave Processor equipped with the True-To-Power power control system. The oven operates at $2.45 \mathrm{GHz}$ and has variable power up to $1000 \mathrm{~W}$. The True-To-Power control system adjusts the power of the magnetron source in order to regulate the temperature of the sample. The oven was modified to allow continuous monitoring of the sample thermal profile. An opening was bored in the upper cavity of the oven and a wire mesh grid placed on the inside of that opening, while a germanium window was placed outside that opening to prevent microwaves from escaping. A FLIR A35SC infrared camera mounted over the germanium window allows for real-time thermal imaging of the sample under microwave exposure (Figure 1).

The compact microwave source shown in Figure 2 has a high frequency signal generator driving a $K_{\alpha}$ band $(29.68 \mathrm{GHz})$ power amplifier. This amplifier feeds the output signal into an open-ended waveguide with an aperture of $1 / 8^{\prime \prime}(3.175 \mathrm{~mm})$ by $3 / 8^{\prime \prime}(9.525 \mathrm{~mm})$, allowing for continuous monitoring of both transmissive and reflective powers. The entire microwave system, that is, the signal generator and the high power amplifier, is the size of a small box. The temperatures were measured with an Infracon Thermal System and a FLIR thermal camera. Composites were exposed to a $10 \mathrm{~W}$ focused microwave beam directed from an open-ended waveguide placed approximately $0.80 \mathrm{~mm}$ from the sample.

For all microwave experiments, the sides of the composites are masked with $3 \mathrm{M}$ metal tape to prevent electrical arching along the edges. Indentations were intentionally formed on several composites using a Future Tech Corp Model FM-7 Vicker's Microhardness Tester with $1 \mathrm{~kg}$ force. Composite samples were impacted with an IMATEK impact tester using $1.13 \mathrm{~kg}(2.5 \mathrm{lbs})$ of weight and drop heights of 5 and 10 inches, corresponding to forces of $1.15 \mathrm{~J}(0.85 \mathrm{ft} \cdot \mathrm{lb})$ and $2.25 \mathrm{~J}$ (1.66 ft.lb), respectively. Composite damage was evaluated by optical microscopy using a Zeiss Axio upright microscope and a Zeiss Axiovert $200 \mathrm{M}$ inverted microscope. Scanning electron images were captured with a Zeiss Supra 55 VP microscope. Indentations and microcracks were identified and copper tape markers were placed near the damage to allow for reidentification of these areas after healing.

\section{Results}

Composites containing SWCNTs, MWCNTs, and spun CNT ribbons show rapid and uniform heating upon exposure to microwaves. Composite panels consisting of spun CNT nanoribbon tend to heat more rapidly and uniformly, which is attributed to higher concentration and better distribution of CNTs throughout the composite. To compare the heating efficacy between CNT-containing composites and composite without CNTs (control samples), two panels were simultaneously exposed to identical microwave conditions. The control panel and the panel containing spun CNT ribbon

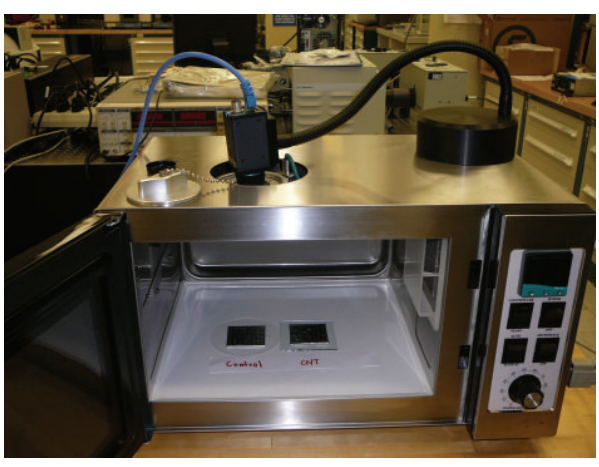

FIGURE 1: Customized microwave oven that allows for controlled heating of samples with in situ thermal profile imaging. Microwave oven is manual or computer controlled and equipped with an optical pyrometer that acts as a feedback loop to provide regulation of the magnetron source and manage sample temperature. An IR camera is mounted onto a window bored into the top of the oven to allow thermal imaging of the sample under microwave exposure.

were irradiated side-by-side and their thermal profiles were captured with the FLIR camera. It was clearly evident that the CNT-containing panel heated more rapidly and uniformly to an elevated temperature of approximately $110^{\circ} \mathrm{C}$ (Figure 3 ). During microwave exposures of these composites, it is observed that the panels heat more quickly at the edges and the thermal gradient moves radially towards the center. The same CNT-containing panel was heated to $100^{\circ} \mathrm{C}$ using $40 \%$ of the $1000 \mathrm{~W}$ capacity of the microwave oven and maintained at this temperature for a duration of 1 hour through regulation of the power setting. As the temperature approached $100^{\circ} \mathrm{C}$, the power level was gradually reduced between 10-15\%, allowing for a uniform and equilibrated temperature of the panel over this period of time. Microwave experiments therefore show that CNTs can facilitate the heating of composites and provide uniform thermal profile over the area of the panel.

The through-thickness heating of the panel is also of interest since repair of internal damage will depend on how well heating translates through the composite thickness. To determine this, a composite was heated and the temperature of the back side was monitored. After reaching a uniform $95^{\circ} \mathrm{C}$ on the front side of the sample, the microwave was powered off and the composite panel was quickly turned over to observe the temperature profile on the back side. The temperature of the back side of the panel was approximately the same and uniform. A piece of metal tape-covered cardboard was placed in the center of a CNT-containing composite to prevent microwave absorption in this area. After exposing the composite to microwave heating, the cardboard piece is removed and the thermal profile of the area underneath is inspected. The thermal image shows the same temperature profile as the surrounding area that was directly exposed to microwaves (Figure 4). It is believed that subsurface heating is induced by exposed CNTs, and this heat is laterally translated to the unexposed area underneath the masking cardboard. Future studies will examine the absorption, reflectance, and transmission of microwaves in 

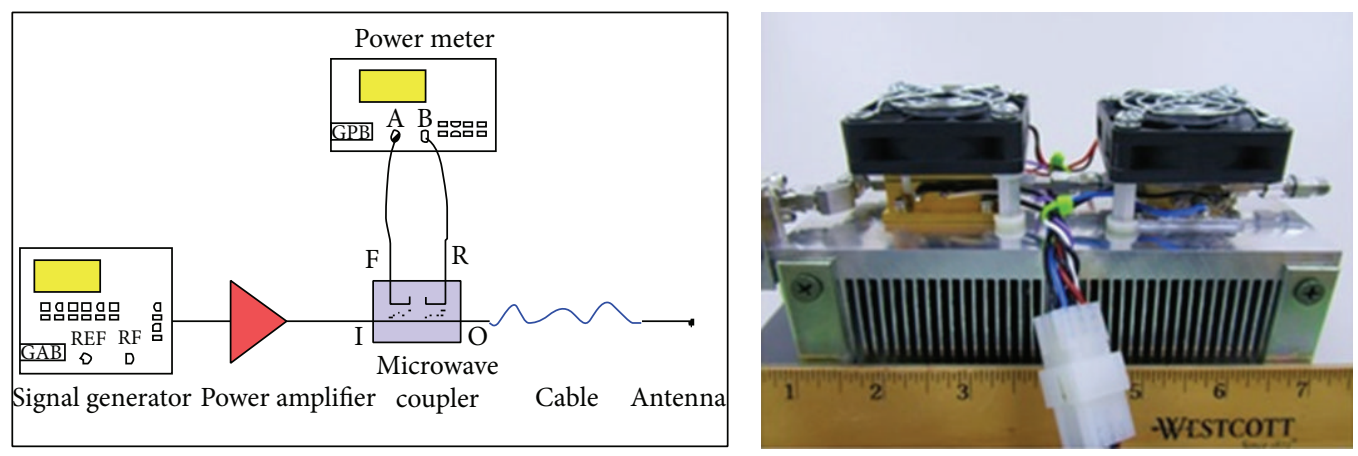

FIGURE 2: A compact microwave generator used to supply focused microwaves through an open end wave guide. The schematic shows the electrical configuration of the entire system used to supply directed microwaves.

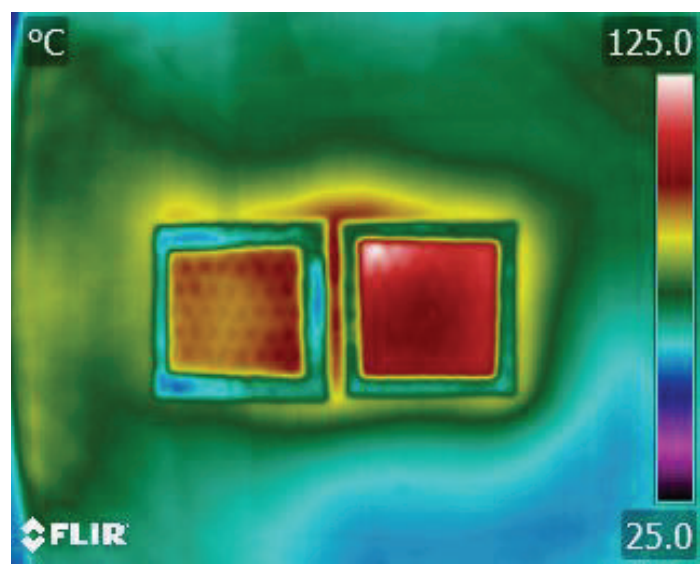

FIGURE 3: Microwave exposure of a composite panel without nanotubes (left) and a nanotube containing composite (right). Thermal imaging indicates more rapid and uniform heating as a result of nanotube incorporation.

these composites to determine the penetration depth of microwaves.

Another premise of microwave heating is that use of a focused microwave source can lead to localized heating of the composites. To prove this, a wave-guided microwave generator was used to irradiate samples. Composites were placed approximately $0.8 \mathrm{~mm}$ beneath the open end of the waveguide, and the temperature was monitored in real time with the FLIR A35SC infrared camera. Figure 5 shows localized heating of CNT-containing panel under microwave exposure. Both the control panel and CNT-containing panel exhibited localized heating; however, panels with CNTs heated much more rapidly to higher temperatures. A timetemperature study was conducted to determine the time required to heat composites to the required temperature for healing. A CNT-containing panel was exposed to microwaves and the temperature was monitored and recorded every $5 \mathrm{sec}-$ onds. It was observed that CNT-containing panels could be locally heated between $90-105^{\circ} \mathrm{C}$ in as little as $15-20$ seconds.

The first set of composite healing experiments was focused on the determination of the minimal temperature required to heal damage within the panel. Previous work on CNT-containing panels showed that the glass transition temperature of composites varied based on the method of CNT inclusion [20]. Direct dispersion of SWCNTs into the polymer matrix lowers the glass transition from $80^{\circ} \mathrm{C}$ to $60^{\circ} \mathrm{C}$. Therefore, various composite panels were heated from $60-100^{\circ} \mathrm{C}$ in increments $10^{\circ} \mathrm{C}$. Three composites containing MWCNT-coated fibers, dispersed SWCNTs, and CNT nanoribbon-coated fibers were indented with a microhardness tester. The panels were incrementally heated to a set temperature for 10 minutes and then inspected via optical microscopy after each heat cycle to view damage healing. The lowest optimal temperature for damage healing was observed to be $95^{\circ} \mathrm{C}$ for all panels.

After establishing the minimal temperature for healing, experiments were conducted to establish the healing efficacy of composites. In this work the healing efficiency was assessed by visual inspection of the healing process. Evaluation of the extent of healing within composites subjected to the same damage conditions and duration of microwave heating was used as a qualitative measure of the healing efficiency. Therefore, a greater extent of damage repair occurring over the same period of microwave heating is interpreted as a higher healing efficiency. Additionally, a shorter period of microwave heating to remove the same extent of inflicted damage is interpreted as a greater healing efficiency. The first of these experiments was to show that CNT inclusion facilitates the healing process. A control panel and a panel containing CNT nanoribbons were intentionally indented, and copper tape markers were used to identify the location of these indentations. The two panels were set side-by-side in the oven and heated with the same microwave power $(15 \%-40 \%)$ for a total of 10 minutes. The CNT-containing panel reached $95^{\circ} \mathrm{C}$ at about 5 minutes into the heating cycle while the control sample reached this temperature only in its corners (Figure 3). Once the CNT panel reached this temperature, the oven power was regulated to maintain that temperature. The copper markers display a different thermal profile which is attributed to difference in emissivity. When the composites were imaged under the optical microscope, complete healing was observed in the CNT panel but not in the control since the control did not reach sufficient temperature. Therefore, it is clear that CNT incorporation improves the efficiency of healing by providing more effective heating. 

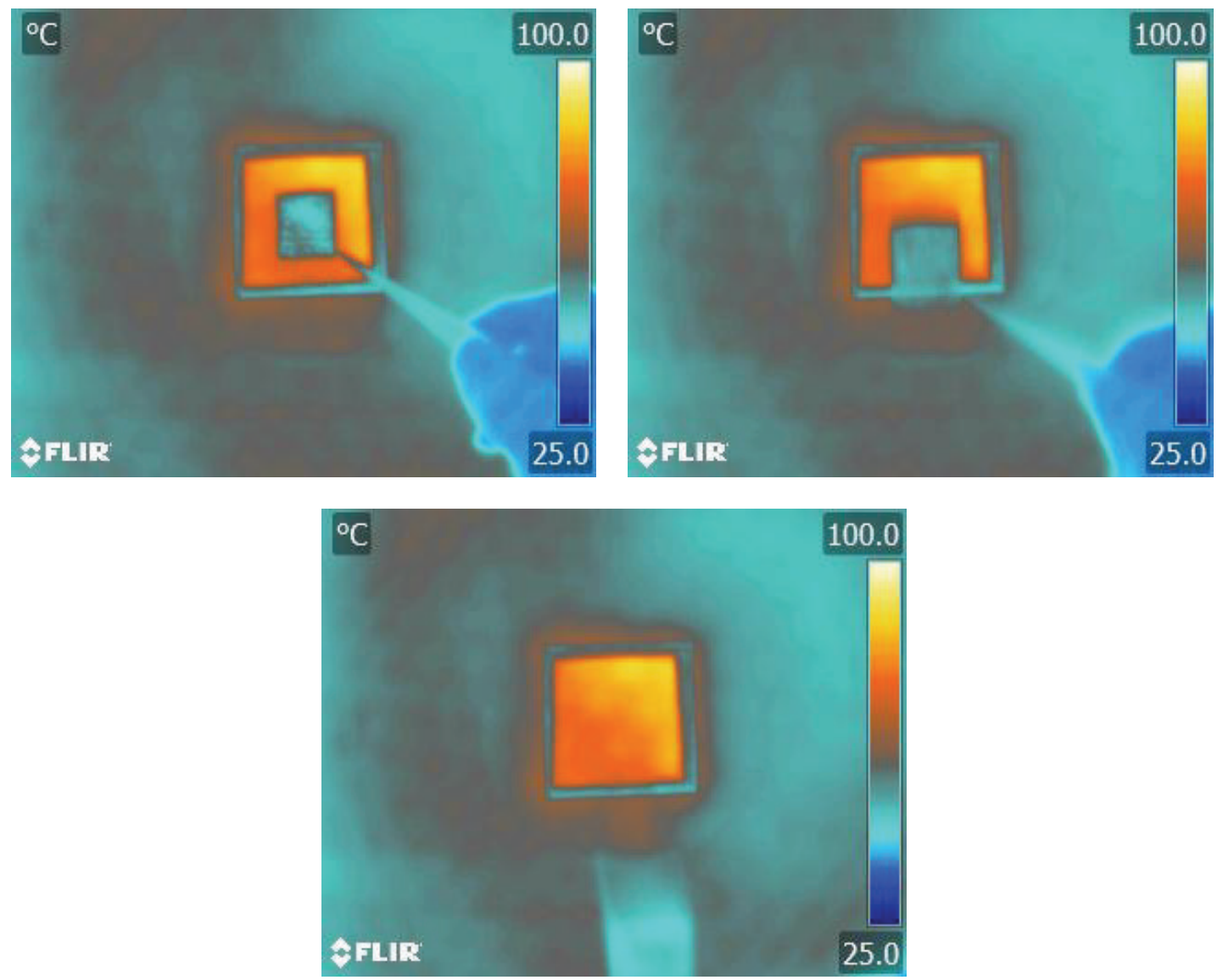

FIGURE 4: Heating of composite panels with the center masked by a piece of metal covered cardboard in which removal of cardboard shows that the area underneath is the same temperature as exposed area. This is attributed to subsurface heating and thermal conductivity of CNTs which allows translation of heat to unexposed area.

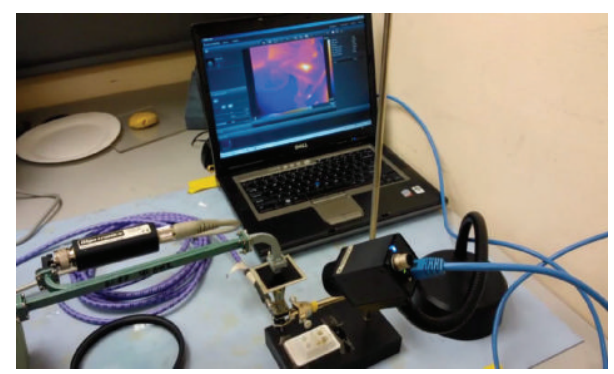

FIGURE 5: Localized heating of a composite panel subjected to $10 \mathrm{~W}$ focused microwaves from an open ended wave guide. Thermal image captured with IR camera displayed on laptop computer shows heating of area directly beneath wave guide. Composite can be heated to over $100^{\circ} \mathrm{C}$ in less than 20 seconds.

To further establish the healing efficacy of composites, heating experiments were conducted to determine the minimal time required for healing. From the previously described experiment, it was found that 5 minutes of heating at $95^{\circ} \mathrm{C}$ was sufficient to heal indents on a CNT panel. The same composite containing CNT nanoribbon was again indented and then heated for 1 and 3 minutes. The composite was indented prior to each heat cycle and inspected via optical microscopy. At 50\% power, the composite could be heated to $\sim 95^{\circ} \mathrm{C}$ in 1.5 minutes after which the power was regulated to keep the temperature constant for an additional 1 and 3 minutes. Partial indentation was still observed after 1 minute of heating while complete healing was observed after 3 minutes. Once minimal heating time was determined, composites containing different forms of CNTs were heated for 3 minutes to compare the healing process. Significant difference was observed in the healing of a composite panel that consisted of SWCNTs dispersed directly into the polymer resin prior to infiltration. In this panel, only partial healing was observed when heated for the same amount of time as compared to the panel containing only nanoribbon-coated fibers (Figure 6). The slower healing rate is attributed to an increase in the viscosity of the polymer due to SWCNT dispersion. The increased viscosity is believed to reduce polymer mobility during healing. The composite, however, was completely healed in 5 minutes when heated to $110^{\circ} \mathrm{C}$. Research has shown that covalent bonding can occur between CNTs and both maleimide and furan compounds [21], which could also be the reason for slower heating rates and higher heating requirements. Further studies will be required to determine the whether this is attributed to viscosity or CNT 

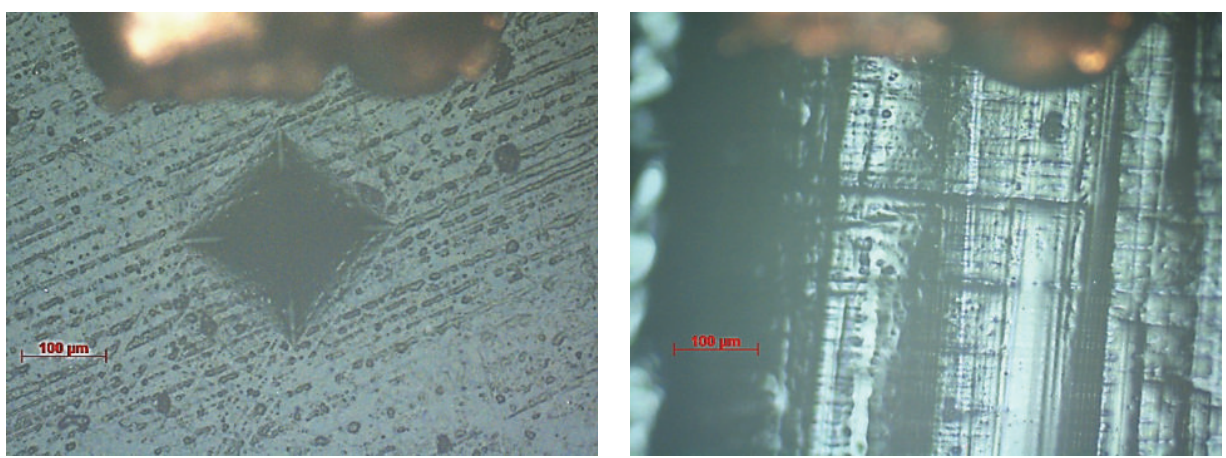

(a)
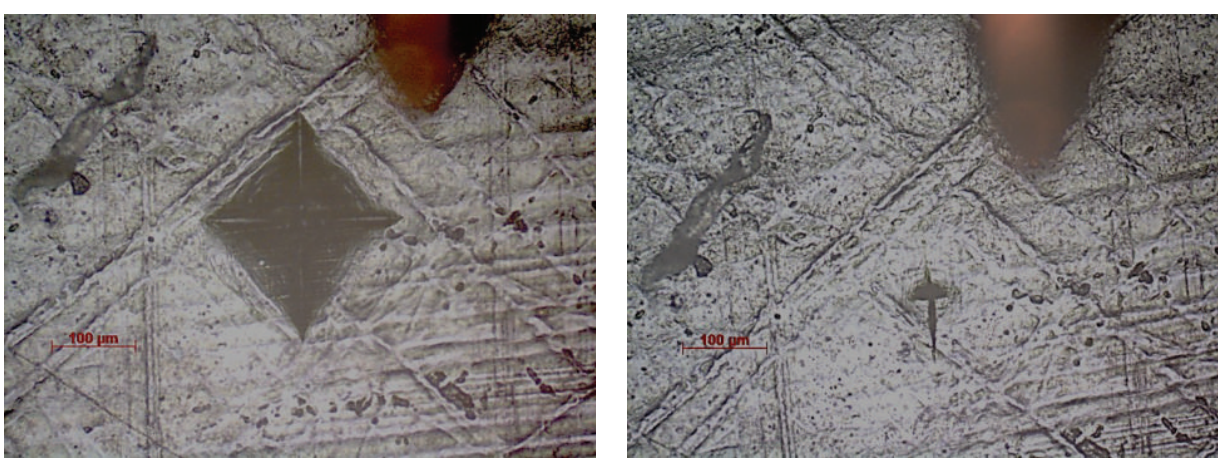

(b)

FIGURE 6: Images of healing in composite panel contacting nanoribbons (a) and panel containing dispersed SWCNTs in polymer matrix (b). Panels were heated to $95^{\circ} \mathrm{C}$ for 3 minutes. SWCNT panel exhibits partial healing and complete healing is achieved by heating to higher temperature.
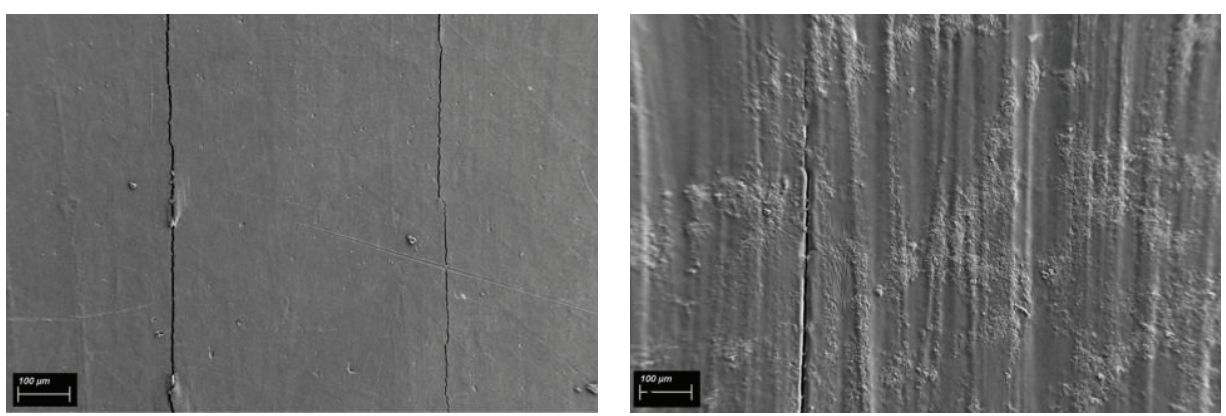

FIGURE 7: SEM images of impacted composite before and after 5 minutes of microwave assisted healing. Near complete healing of crack on right is observed while partial healing of wider crack is observed. The smooth appearance of the coupon's surface, induced by the mold during composite fabrication, is not retained posthealing.

chemical interactions with the polymer resin. In either case, healing is readily achievable in all CNT composites, requiring only a few minutes to repair damage.

A composite panel containing MWCNT-coated carbon fibers was impacted and heated in order to heal damage. Prior to heating, both sides of the panel were inspected for damage by optical and scanning electron microscopy. Cracks were observed on the front and back sides of the composite panels, and copper tape markers were placed on both sides to enable reidentification of damaged areas. The composite was heated to $95^{\circ} \mathrm{C}$ for 5 minutes with the impacted side of facing up. It was also observed that opposite sides of impacted composite panels heal at different rates, with the underside healing more rapidly than the top side. Wider cracks showed partial healing, while thinner cracks exhibited nearly complete healing (Figure 7). It is thought that polymer mobility from top to bottom through the panel thickness is responsible for this effect. Since these composites are under study towards their application to human spaceflight in both vacuum and the microgravity environment, the effects of gravity and pressure on healing capability and efficiency are germane and therefore targeted for future studies. It is anticipated that this disparity of healing rates between the top side and underside will be resolved in the microgravity 
environment. Depending on the extent of damage, longer healing times may be required to properly heal damage. Future studies will focus on the orientation and pressure effects regarding healing efficacy of composites.

\section{Conclusion}

Incorporation of carbon nanotubes into composites capable of thermal reversible polymerization can be effectively heated by microwave exposure. CNTs provide rapid and uniform heating of the composite to the temperature required for inducing thermally activated healing. Heating is believed to be subsurface since unexposed areas and the backside to the composite reach the same temperature. The enhanced thermal conductivity of CNT may also contribute to this observation. This effect is highly desirable since this could provide a means for microwave assisted curing of composites or repair of manufacturing induced defects. Additionally, the introduction of CNTs into composites allows localized heating when a focused microwave source is used. Localized heating may have many advantages since only the area that requires healing will be subjected to softening. When composite are exposed to a focused microwave source the exposed area can be heated to above $100^{\circ} \mathrm{C}$ in less than 20 seconds.

The healing efficacy of composites is significantly improved by the incorporation of CNTs. Intentionally damaged composites can be healed in as little as 3 minutes depending on the extent of damage and the means in which CNTs are introduced into composites. Dispersion of CNT directly into the polymer resin results in a longer healing time when heated to the same temperature compared to composites with only CNT carbon fiber coatings. This is believed to be attributed to either an increase in the viscosity of the polymer resin or direct bonding between CNTs and polymer resin. Composites with CNTs dispersed into the resin require slightly higher temperatures to achieve healing in the same period of time. It was also observed than different sides of impacted composites heal at dissimilar rates. The effect of gravity on polymer mobility is attributed to this observation. Future studies will investigate the effect of gravity and pressure on the healing ability of these composites. Composites will be placed under compressive and tensile loads to examine the influence of pressure on composite healing.

\section{Conflict of Interests}

The authors declare that there is no conflict of interests regarding the publication of this paper.

\section{Acknowledgments}

The authors would like to thanks Dr. Anvar Zhakidov, Dr. John Ferraris, and William Holmes at Solarno for the deposition of spun carbon nanotubes onto carbon fibers. Authors would also like to thank Dr. Dickey Arndt at the Johnson Space Center for use of the compact waveguided microwave source for localized heating experiments. This work was funded through the NASA Center Innovation Fund directed by the Johnson Space Center towards Independent Research and Development. This paper is based on work supported by the Air Force Office of Scientific Research Contract FA9550-11-C-0008. Appreciation is extended to Dr. B. Les Lee, Program Manager, for his encouragement and support.

\section{References}

[1] G. Marsh, "Boeing's 787: trials, tribulations, and restoring the dream," Reinforced Plastics, vol. 53, no. 8, pp. 16-21, 2009.

[2] Boeing, "design highlights/advanced-composite-use".

[3] M. R. Kessler, "Self-healing: a new paradigm in materials design," Proceedings of the Institution of Mechanical Engineers, Part G: Journal of Aerospace Engineering, vol. 221, no. 4, pp. 479495, 2007.

[4] D. Y. Wu, S. Meure, and D. Solomon, "Self-healing polymeric materials: a review of recent developments," Progress in Polymer Science, vol. 33, no. 5, pp. 479-522, 2008.

[5] S. D. Bergman and F. Wudl, "Mendable polymers," Journal of Materials Chemistry, vol. 18, no. 1, pp. 41-62, 2008.

[6] J. Hu, H.-Q. Chen, and Z. Zhang, "Mechanical properties of melamine formaldehyde microcapsules for self-healing materials," Materials Chemistry and Physics, vol. 118, no. 1, pp. 63-70, 2009.

[7] J. W. C. Pang and I. P. Bond, "A hollow fibre reinforced polymer composite encompassing self-healing and enhanced damage visibility," Composites Science and Technology, vol. 65, no. 11-12, pp. 1791-1799, 2005.

[8] A. R. Jones, B. J. Blaiszik, S. R. White, and N. R. Sottos, "Full recovery of fiber/matrix interfacial bond strength using a microencapsulated solvent-based healing system," Composites Science and Technology, vol. 79, pp. 1-7, 2013.

[9] B. J. Blaiszik, M. M. Caruso, D. A. McIlroy, J. S. Moore, S. R. White, and N. R. Sottos, "Microcapsules filled with reactive solutions for self-healing materials," Polymer, vol. 50, no. 4, pp. 990-997, 2009.

[10] A. R. Hamilton, N. R. Sottos, and S. R. White, "Mitigation of fatigue damage in self-healing vascular materials," Polymer, vol. 53 , no. 24 , pp. 5575-5581, 2012.

[11] A. M. Coppola, P. R. Thakre, N. R. Sottos, and S. R. White, "Tensile properties and damage evolution in vascular 3D woven glass/epoxy composites," Composites Part A: Applied Science and Manufacturing, vol. 59, pp. 9-17, 2014.

[12] X. Chen, F. Wudl, A. K. Mal, H. Shen, and S. R. Nutt, "New thermally remendable highly cross-linked polymeric materials," Macromolecules, vol. 36, no. 6, pp. 1802-1807, 2003.

[13] F. Ghezzo, D. R. Smith, T. N. Starr et al., "Development and characterization of healable carbon fiber composites with a reversibly cross linked polymer," Journal of Composite Materials, vol. 44, no. 13, pp. 1587-1603, 2010.

[14] Y. Amamoto, J. Kamada, H. Otsuka, A. Takahara, and K. Matyjaszewski, "Repeatable photoinduced self-healing of covalently cross-linked polymers through reshuffling of trithiocarbonate units," Angewandte Chemie, vol. 50, no. 7, pp. 1660-1663, 2011.

[15] T. F. Scott, A. D. Schneider, W. D. Cook, and C. N. Bowman, "Photoinduced plasticity in cross-linked polymers," Science, vol. 308, no. 5728, pp. 1615-1617, 2005. 
[16] A. Rekondo, R. Martin, A. Ruiz de Luzuriaga, G. Cabañero, H. J. Grande, and I. Odriozola, "Catalyst-free room-temperature self-healing elastomers based on aromatic disulfide metathesis," Materials Horizons, vol. 1, pp. 237-240, 2014.

[17] J. S. Park, T. Darlington, A. F. Starr, K. Takahashi, J. Riendeau, and H. T. Hahn, "Multiple healing effect of thermally activated self-healing composites based on Diels-Alder reaction," Composites Science and Technology, vol. 70, no. 15, pp. 2154-2159, 2010.

[18] K. R. Paton and A. H. Windle, "Efficient microwave energy absorption by carbon nanotubes," Carbon, vol. 46, no. 14, pp. 1935-1941, 2008.

[19] T. J. Imholt, C. A. Dyke, B. Hasslacher et al., "Nanotubes in microwave fields: light emission, intense heat, outgassing and reconstruction," Chemistry of Materials, vol. 15, no. 21, pp. 39693970, 2003.

[20] E. D. Sosa, T. K. Darlington, B. A. Hanos, and M. J. E. O’Rourke, "Multifunctional thermally remendable nanocomposites," Journal of Composites, vol. 2014, Article ID 705687, 12 pages, 2014.

[21] C.-M. Chang and Y.-L. Liu, "Functionalization of multi-walled carbon nanotubes with furan and maleimide compounds through Diels-Alder cycloaddition," Carbon, vol. 47, no. 13, pp. 3041-3049, 2009. 

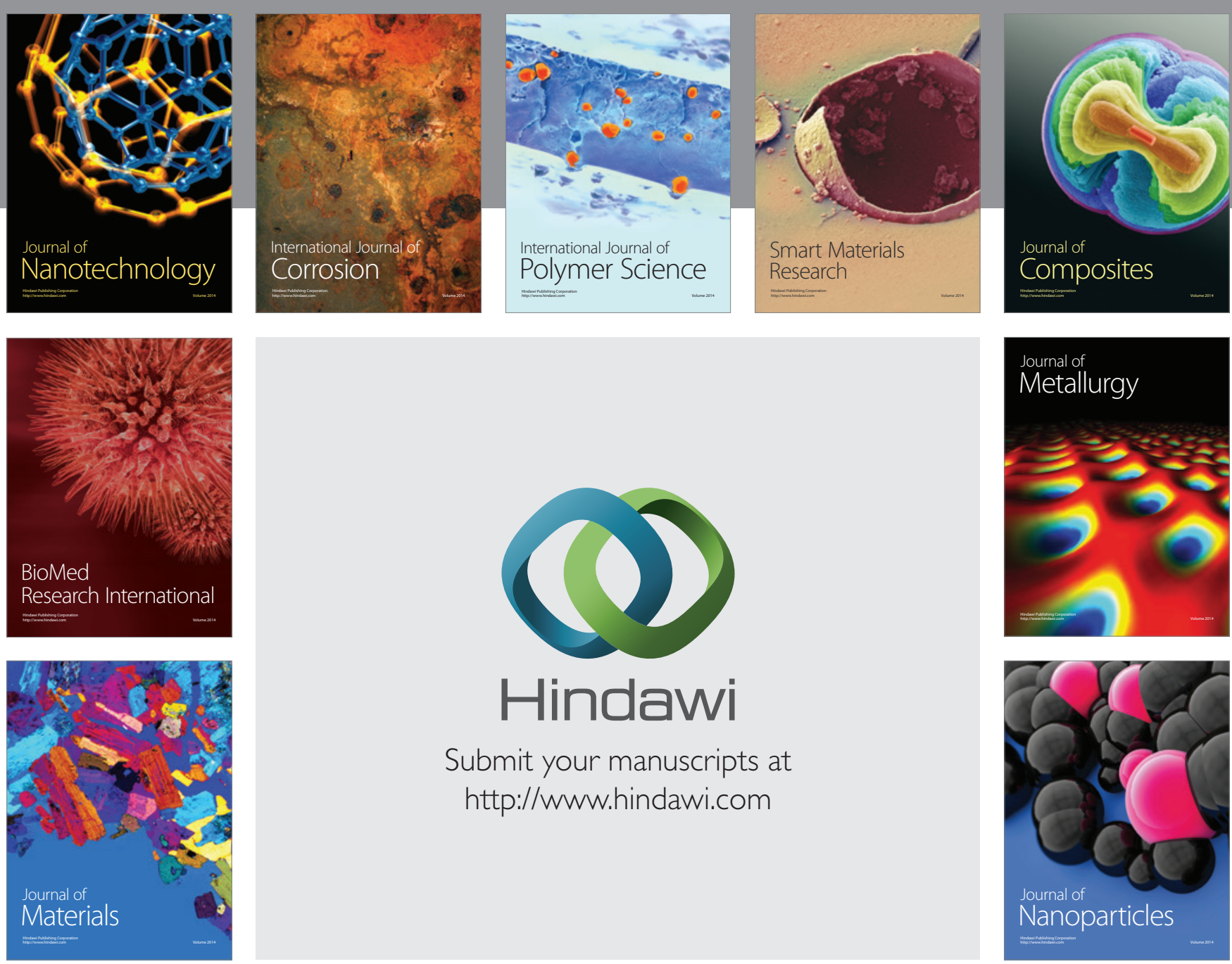

Submit your manuscripts at http://www.hindawi.com
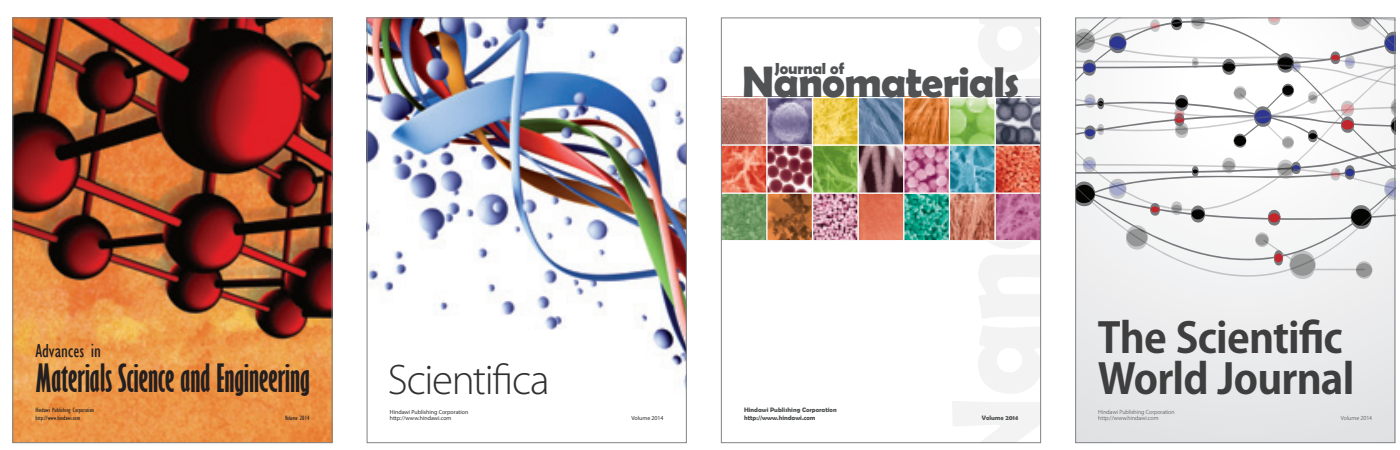

\section{The Scientific World Journal}
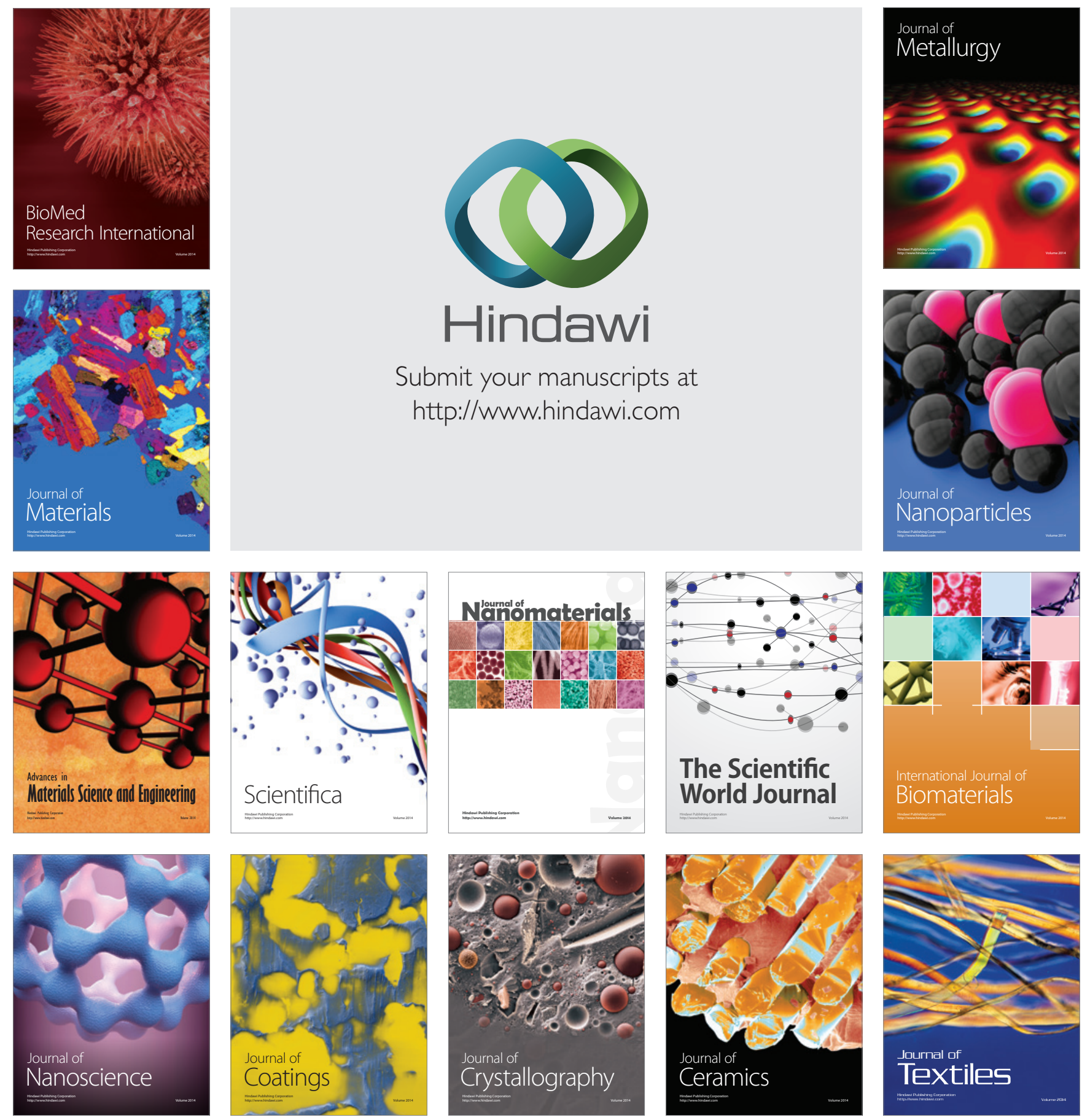